\title{
Studying Two-body Nonleptonic Weak Decays of Hyperons with Topological Diagram Approach
}

\author{
Yuan-Guo $\mathrm{Xu}^{1, \dagger}$, Xiao-Dong Cheng ${ }^{2, \ddagger}$, Jie-Lei Zhang, ${ }^{2, \sharp}$ and Ru-Min Wang ${ }^{1, \S}$ \\ ${ }^{1}$ College of Physics and Communication Electronics, JiangXi Normal University, NanChang, JiangXi 330022, China \\ ${ }^{2}$ College of Physics and Electronic Engineering, XinYang Normal University, XinYang, Henan 464000, China \\ $\dagger$ yuanguoxv@163.com $\quad{ }^{\ddagger}$ chengxd@mails.ccnu.edu.cn

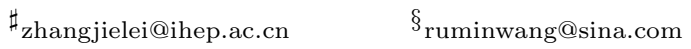

\begin{abstract}
Many decays of light baryons consisting of light $u, d, s$ quarks have been measured, and these measurements will help us to understand the decay properties of light baryons. In this work, we study two-body nonleptonic weak decays of light baryon octet $\left(T_{8}\right)$ and baryon decuplet $\left(T_{10}\right)$ by the topological diagram approach (TDA) under the SU(3) flavor symmetry for the first time. We find that (1) the TDA and the SU(3) irreducible representation approach (IRA) match consistently in $T_{10} \rightarrow T_{8,10}^{\left(^{\prime}\right)} P_{8}\left(P_{8}\right.$ is the light pseudoscalar meson octet); (2) almost all relevant not-yet-measured $\mathcal{B}\left(T_{10} \rightarrow T_{8} P_{8}\right)$ may be predicted by using three experimental data of $\mathcal{B}\left(\Omega^{-} \rightarrow \Xi^{0} \pi^{-}, \Xi^{-} \pi^{0}, \Lambda^{0} K^{-}\right)$, and the upper limits of $\mathcal{B}\left(T_{10} \rightarrow T^{\prime}{ }_{10} \pi^{-}\right)$may be obtained from the experimental upper limit of $\mathcal{B}\left(\Omega^{-} \rightarrow \Xi^{* 0} \pi^{-}\right)$by both the TDA and the IRA together, nevertheless, all new predicted branching ratios are too small to br reached in current experiments; (3) $T_{8} \rightarrow T_{8}^{\prime} P_{8}$ decays are quite complex in terms of the TDA, and we find that W-exchange diagrams give large and even dominant contributions by using relevant experimental data and the isospin relations.
\end{abstract}

\section{INTRODUCTION}

Many two-body nonleptonic weak decays of the octet and decuplet baryons (such as $\Sigma^{+} \rightarrow p \pi^{0}, \Sigma^{+} \rightarrow n \pi^{+}$, $\Sigma^{-} \rightarrow n \pi^{-}, \Lambda^{0} \rightarrow p \pi^{-}, \Lambda^{0} \rightarrow n \pi^{0}, \Xi^{-} \rightarrow \Lambda^{0} \pi^{-}, \Xi^{0} \rightarrow \Lambda^{0} \pi^{0}, \Omega^{-} \rightarrow \Xi^{0} \pi^{-}, \Xi^{-} \pi^{0}, \Lambda^{0} K^{-}$) were measured a long time ago by SPEC, HBC, OSPK etc [1]. Now the sensitivity for measurements of $\Lambda, \Sigma, \Xi, \Omega$ hyperon decays is in the range of $10^{-5}-10^{-8}$ at the BESIII [2-5], and these hyperons are also produced copiously at the LHCb experiment $[6,7]$. The precise measurements from the BESIII and LHCb experiments will confirm the earlier experimental data from SPEC, HBC, OSPK etc, and will give new information about determining the V-A structure and quark-flavor mixing [8-10] as well as probing the non-standard charged current interactions [11, 12].

In the theoretical side, the heavy quark expansion can not be used in the light baryon decays, and the factorization does not work well. There is no reliable method to calculate these decay matrix elements at present. In the lack of reliable calculations, some model-independent approaches can provide very useful information about the decays, such as $\mathrm{SU}(3) / \mathrm{U}(3)$ flavor symmetry (see for instance Refs. [13-29]) and flavor topological diagram approach (TDA) (see for instance Refs. [30-44]). These approaches are independent of the detailed dynamics, and offer an opportunity to relate different decay modes. The decay matrix elements are directly extracted from the experimental data, despite of their unclear sources. In the TDA, decay amplitudes are represented by connecting quark line flows in different ways and then relate them by the SU(3) symmetry, therefore, the TDA gives a better understanding of dynamics in the different amplitudes. 
The related decays have also been extensively studied, for instance, in Refs. [45-57]. The studies in Refs. [10, 28, 5861] show that the SU(3) flavor symmetry breaking effects are small in the semileptonic hyperon decays and the nonleptonic hyperon two-body weak decays. So, in this paper, we will study $T_{10} \rightarrow T_{8} M_{8}, T_{10} \rightarrow T_{10}^{\prime} M_{8}$ and $T_{8} \rightarrow T_{8}^{\prime} M_{8}$ nonleptonic two-body weak decays by using the TDA under the SU(3) flavor symmetry, which is the following up work of Ref. [28]. We will firstly construct the TDA amplitudes for different kinds of $T_{8}$ and $T_{10}$ nonleptonic decays, secondly obtain the decay amplitude relations between different decay modes, then extract the TDA amplitudes from the available data, and finally analyze the size of the different kinds of contributions or predict the not-yet-measured modes for further tests in experiments.

This paper is organized as follows. In Sec. II, we give the effective Hamiltonian for nonleptonic $s \rightarrow u \bar{u} d$ process and the expression of the branching ratios. In Sec. III, we firstly give the TDA amplitudes and then give our numerical results and analyses for $T_{10} \rightarrow T_{8} M_{8}, T_{10} \rightarrow T_{10}^{\prime} M_{8}$ and $T_{8} \rightarrow T_{8}^{\prime} M_{8}$ decays. Section IV contains our summary and conclusion.

\section{Theoretical framework}

Under the $S U(3)$ flavor symmetry of $u, d, s$ quarks, the light baryon octet $T_{8}$, the light baryon decuplet $T_{10}$, the light pseudoscalar meson octet $P_{8}$, and the vector meson octet $V_{8}$ can be written as

$$
\begin{aligned}
T_{8}= & \left(\begin{array}{ccc}
\frac{\Lambda^{0}}{\sqrt{6}}+\frac{\Sigma^{0}}{\sqrt{2}} & \Sigma^{+} & p \\
\Sigma^{-} & \frac{\Lambda^{0}}{\sqrt{6}}-\frac{\Sigma^{0}}{\sqrt{2}} & n \\
\Xi^{-} & \Xi^{0} & -\frac{2 \Lambda^{0}}{\sqrt{6}}
\end{array}\right), \\
T_{10}= & \frac{1}{\sqrt{3}}\left(\left(\begin{array}{ccc}
\sqrt{3} \Delta^{++} & \Delta^{+} & \Sigma^{*+} \\
\Delta^{+} & \Delta^{0} & \frac{\Sigma^{* 0}}{\sqrt{2}} \\
\Sigma^{*+} & \frac{\Sigma^{* 0}}{\sqrt{2}} & \Xi^{* 0}
\end{array}\right),\left(\begin{array}{ccc}
\Delta^{+} & \Delta^{0} & \frac{\Sigma^{* 0}}{\sqrt{2}} \\
\Delta^{0} & \sqrt{3} \Delta^{-} & \Sigma^{*-} \\
\frac{\Sigma^{* 0}}{\sqrt{2}} & \Sigma^{*-} & \Xi^{*-}
\end{array}\right),\left(\begin{array}{ccc}
\Sigma^{*+} & \frac{\Sigma^{* 0}}{\sqrt{2}} & \Xi^{* 0} \\
\frac{\Sigma^{* 0}}{\sqrt{2}} & \Sigma^{*-} & \Xi^{*-} \\
\Xi^{*-} & \Xi^{*-} & \sqrt{3} \Omega^{-}
\end{array}\right)\right), \\
P_{8}= & \left(\begin{array}{ccc}
\frac{\eta_{8}}{\sqrt{6}}+\frac{\pi^{0}}{\sqrt{2}} & \pi^{+} & K^{+} \\
\pi^{-} & \frac{\eta 8}{\sqrt{6}}-\frac{\pi^{0}}{\sqrt{2}} & K^{0} \\
K^{-} & \bar{K}^{0} & -\sqrt{\frac{2}{3}} \eta_{8}
\end{array}\right), \\
V_{8}= & \left(\begin{array}{ccc}
\frac{\omega_{8}}{\sqrt{6}}+\frac{\rho^{0}}{\sqrt{2}} & \rho^{+} & K^{*+} \\
\rho^{-} & \frac{\omega_{8}}{\sqrt{6}}-\frac{\rho^{0}}{\sqrt{2}} & K^{* 0} \\
K^{*-} & \bar{K}^{* 0} & -\sqrt{\frac{2}{3}} \omega_{8}
\end{array}\right) .
\end{aligned}
$$

Two-body nonleptonic weak decays $T_{8,10} \rightarrow T_{8,10}^{\prime} M_{8}$ with $M_{8}=P_{8}, V_{8}$ are induced by $s \rightarrow u \bar{u} d$ transition. As given in Ref. [28], there are two kinds of diagrams for s-quark weak decay in the Standard Model, the tree level diagram and the penguin diagram. The effective Hamiltonian for nonleptonic $s \rightarrow u \bar{u} d$ process at scales $\mu<m_{c}$ can be written as [62]

$$
\mathcal{H}_{e f f}=\frac{G_{F}}{\sqrt{2}} V_{u d} V_{u s}^{*} \sum_{i=1}^{10}\left[z_{i}(\mu)-\frac{V_{t d} V_{t s}^{*}}{V_{u d} V_{u s}^{*}} y_{i}(\mu)\right] Q_{i}(\mu),
$$

where $V_{u q}$ is the CKM matrix element, $z_{i}(\mu)$ and $y_{i}(\mu)$ are Wilson coefficients, and $Q_{i}$ is four-quark operators. The detail can be found in Refs. [28, 62]. 
The relevant topological diagrams for $T_{8,10} \rightarrow T_{8} M_{8}$ nonleptonic weak decays are displayed in Fig. 1 . Since the octet baryon is not fully symmetric or antisymmetric in flavor space, there are more than one amplitudes corresponding to one topological diagram. Noted that Fig. $1(\mathrm{c}, \sigma)$ only contributes the decays with $M_{8}=\eta$, $\omega$, which will not be considered in this work. Other topological diagrams in Fig. 1 can be divided into three categories: the tree diagrams in Fig. 1 (a,b), the W-exchange diagrams in Fig. 1 (d,e,f,g) and the penguin diagrams in Fig. $1(\lambda, \gamma)$. Fig. 1 (a) contributes to the decays with the charged $M_{8}$, which is proportional to the color-flavor factor $C_{2}+\frac{C_{1}}{N_{C}}$. Fig. 1 (b) contributes to the decays with the neutral $M_{8}$, which is proportional to $C_{1}+\frac{C_{2}}{N_{C}}$. The contributions of Fig. 1 (d,e,f,g) are all proportional to $C_{2}-C_{1}$. Compared with the tree diagrams and $\mathrm{W}$-exchange contributions related to $C_{1,2}$, the penguin contributions displayed in Fig. $1(\lambda, \gamma)$ are strongly suppressed by small Wilson coefficients $C_{3, \cdots, 10}$ and quite small CKM factor, and their contributions can be safely ignored in these decays if one do not consider the information of $\mathrm{CP}$ violation.

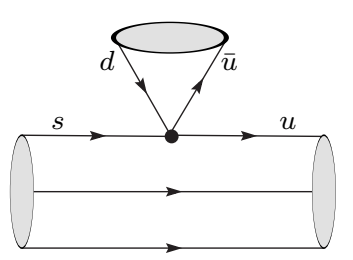

$$
\begin{aligned}
& T_{10} \rightarrow T_{8} M_{8}: \hat{a}_{1} \\
& T_{10} \rightarrow T_{10}^{\prime} M_{8}: \check{a} \\
& T_{8} \rightarrow T_{8}^{\prime} M_{8}: \tilde{a}_{1, \cdots, 5}
\end{aligned}
$$

( a )

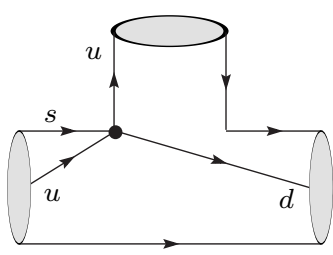

$$
\begin{aligned}
& T_{10} \rightarrow T_{8} M_{8}: \hat{e}_{1,2,3} \\
& T_{10} \rightarrow T_{10}^{\prime} M_{8}: \check{e} \\
& T_{8} \rightarrow T_{8}^{\prime} M_{8}: \tilde{e}_{1, \cdots, 9}
\end{aligned}
$$

( e )

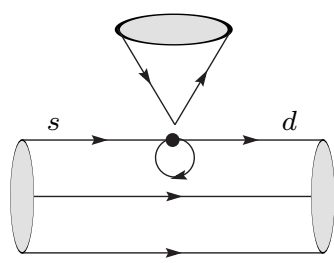

$$
\begin{aligned}
& T_{10} \rightarrow T_{8} M_{8}: \hat{\sigma}_{1} \\
& T_{10} \rightarrow T_{10}^{\prime} M_{8}: \check{\sigma} \\
& T_{8} \rightarrow T_{8}^{\prime} M_{8}: \tilde{\sigma}_{1, \cdots, 5}
\end{aligned}
$$

$(\sigma)$

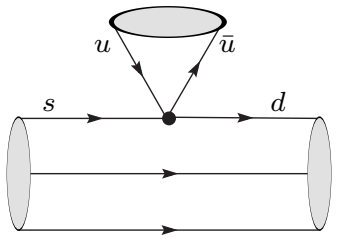

$T_{10} \rightarrow T_{8} M_{8}: \hat{b}_{1}$

$T_{10} \rightarrow T_{10}^{\prime} M_{8}: \check{b}$ $T_{8} \rightarrow T_{8}^{\prime} M_{8}: \tilde{b}_{1, \cdots, 5}$

( b )

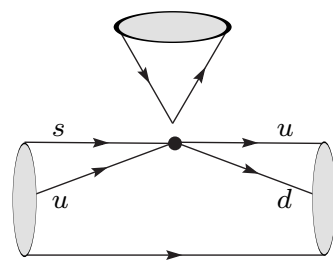

$T_{10} \rightarrow T_{8} M_{8}: \hat{c}_{1,2,3}$

$T_{10} \rightarrow T_{10}^{\prime} M_{8}: \check{c}$

$T_{8} \rightarrow T_{8}^{\prime} M_{8}: \tilde{c}_{1}, \cdots, 9$

(c)

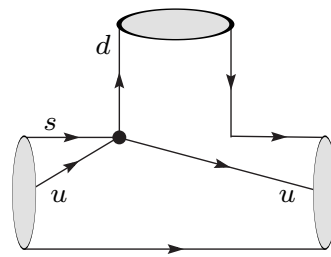

$T_{10} \rightarrow T_{8} M_{8}: \hat{d}_{1,2,3}$

$T_{10} \rightarrow T_{10}^{\prime} M_{8}: \check{d}$

$T_{8} \rightarrow T_{8}^{\prime} M_{8}: \tilde{d}_{1, \cdots, 9}$

(d)

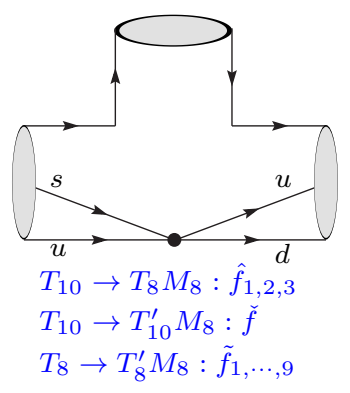

( f )

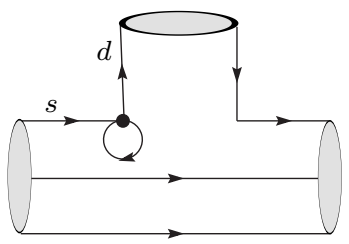

$T_{10} \rightarrow T_{8} M_{8}: \hat{\lambda}_{1}$

$T_{10} \rightarrow T_{10}^{\prime} M_{8}: \check{\lambda}$

$T_{8} \rightarrow T_{8}^{\prime} M_{8}: \tilde{\lambda}_{1, \cdots, 5}$

$(\lambda)$

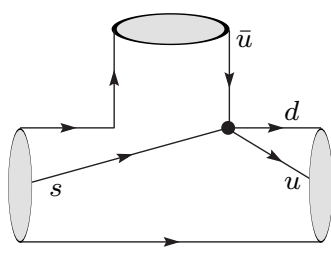

$T_{10} \rightarrow T_{8} M_{8}: \hat{g}_{1,2,3}$

$T_{10} \rightarrow T_{10}^{\prime} M_{8}: \check{g}$

$T_{8} \rightarrow T_{8}^{\prime} M_{8}: \tilde{g}_{1, \cdots, 9}$

( g )

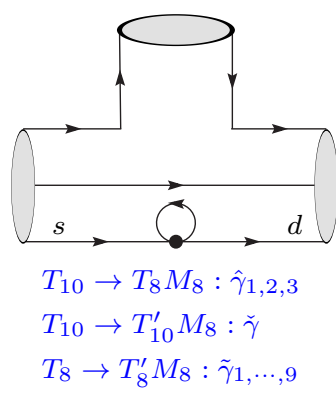

$(\gamma)$

FIG. 1: Topological diagrams for $T_{8,10} \rightarrow T_{8,10}^{\prime} M_{8}$ nonleptonic decays. Since the octed baryon $T_{8}$ is not fully asymmetric or antisymmetric in flavor space, there might be more than one amplitudes corresponding to one relevant topological diagram. 
The branching ratios of the $T_{A} \rightarrow T_{B} M_{C}$ two-body decays can be written in terms of the decay amplitudes $A\left(T_{A} \rightarrow T_{B} M_{C}\right)$

$$
\mathcal{B}\left(T_{A} \rightarrow T_{B} M_{C}\right)=\frac{\tau_{A}\left|p_{c m}\right|}{8 \pi m_{A}^{2}}\left|A\left(T_{A} \rightarrow T_{B} M_{C}\right)\right|^{2} S
$$

where $T_{A, B}=T_{8}$ or $T_{10}, M_{C}=M_{8},\left|p_{c m}\right|=\sqrt{\left(m_{A}^{2}-\left(m_{B}+m_{C}\right)^{2}\right)\left(m_{A}^{2}-\left(m_{B}-m_{C}\right)^{2}\right)} /\left(2 m_{A}\right), S=1$ for $T_{A}=T_{8}$, and $S=1 / 2$ for $T_{A}=T_{10}$. For more accurate results, we will consider the mass difference in $A\left(T_{A} \rightarrow T_{B} P_{8}\right)$ from Eqs. (66-68) of Ref. [63]

$$
A\left(T_{A} \rightarrow T_{B} P_{8}\right) \propto \frac{m_{A}}{m_{B}} p_{c m} N_{B} N_{A}
$$

with $N_{A}=\sqrt{2 m_{A}}$ and $N_{B}=\sqrt{\left(\left(m_{A}+m_{B}\right)^{2}-m_{P}^{2}\right) /\left(2 m_{A}\right)}$. The amplitudes $A\left(T_{A} \rightarrow T_{B} M_{C}\right)$ for different decay processes are given in next section.

\section{Results and discussions}

In this section, we only give the concrete amplitudes of $T_{8,10} \rightarrow T_{8,10}^{\prime} P_{8}$ decays, and the corresponding amplitudes of $T_{8,10} \rightarrow T_{8,10}^{\prime} V_{8}$ decays can be obtained similarly by replacing $P_{8}$ to $V_{8}$ in following Tab. I, Tab. III and Tab. VI. For $T_{8} \rightarrow T_{10} M_{8}$ decays with $s \rightarrow u \bar{u} d$ transition, we find that these processes are not allowed by the energy conservation law. So we will only give the detail analyses for $T_{10} \rightarrow T_{8} P_{8}, T_{10} \rightarrow T_{10}^{\prime} P_{8}$ and $T_{8} \rightarrow T_{8}^{\prime} P_{8}$ weak decays. In addition, the theoretical input parameters and the experimental data within the $1 \sigma$ error from Particle Data Group [1] will be used in our numerical results.

\section{A. $\quad T_{10} \rightarrow T_{8} M_{8}$ weak decays}

Topological diagrams for $T_{10} \rightarrow T_{8} M_{8}$ weak decays are displayed in Fig. 1, and the TDA amplitudes of the $T_{10} \rightarrow T_{8} M_{8}$ weak decays can be written as

$$
\begin{aligned}
A\left(T_{10} \rightarrow T_{8} M_{8}\right)^{T D A} & =\hat{a}_{1} H_{m n}^{\prime k l}\left(T_{10}\right)^{n i j}\left(T_{8}\right)_{[i k] j}\left(M_{8}\right)_{l}^{m} \\
& +\hat{b}_{1} H_{m n}^{\prime k l}\left(T_{10}\right)^{n i j}\left(T_{8}\right)_{[i l] j}\left(M_{8}\right)_{k}^{m} \\
& +\hat{c}_{1} H_{k n}^{\prime i l}\left(T_{10}\right)^{n i j}\left(T_{8}\right)_{[j k] l}\left(M_{8}\right)_{m}^{m}+\hat{c}_{2} H_{k n}^{\prime i l}\left(T_{10}\right)^{n i j}\left(T_{8}\right)_{[j l] k}\left(M_{8}\right)_{m}^{m}+\hat{c}_{3} H_{k n}^{\prime i l}\left(T_{10}\right)^{n i j}\left(T_{8}\right)_{[k l] j}\left(M_{8}\right)_{m}^{m} \\
& +\hat{d}_{1} H_{i n}^{\prime l k}\left(T_{10}\right)^{n i j}\left(T_{8}\right)_{[j m] k}\left(M_{8}\right)_{l}^{m}+\hat{d}_{2} H_{i n}^{\prime l k}\left(T_{10}\right)^{n i j}\left(T_{8}\right)_{[j k] m}\left(M_{8}\right)_{l}^{m}+\hat{d}_{3} H_{i n}^{\prime l k}\left(T_{10}\right)^{n i j}\left(T_{8}\right)_{[k m] j}\left(M_{8}\right)_{l}^{m} \\
& +\hat{e}_{1} H_{i n}^{\prime k l}\left(T_{10}\right)^{n i j}\left(T_{8}\right)_{[j m] l}\left(M_{8}\right)_{k}^{m}+\hat{e}_{2} H_{i n}^{\prime k l}\left(T_{10}\right)^{n i j}\left(T_{8}\right)_{[j l] m}\left(M_{8}\right)_{k}^{m}+\hat{e}_{3} H_{i n}^{\prime k l}\left(T_{10}\right)^{n i j}\left(T_{8}\right)_{[l m] j}\left(M_{8}\right)_{k}^{m} \\
& +\hat{f}_{1} H_{i n}^{\prime k l}\left(T_{10}\right)^{n i j}\left(T_{8}\right)_{[k l] m}\left(M_{8}\right)_{j}^{m}+\hat{f}_{2} H_{i n}^{\prime k l}\left(T_{10}\right)^{n i j}\left(T_{8}\right)_{[k m] l}\left(M_{8}\right)_{j}^{m}+\hat{f}_{3} H_{i n}^{\prime k l}\left(T_{10}\right)^{n i j}\left(T_{8}\right)_{[m l] k}\left(M_{8}\right)_{j}^{m} \\
& +\hat{g}_{1} H_{m n}^{\prime l k}\left(T_{10}\right)^{n i j}\left(T_{8}\right)_{[i k] l}\left(M_{8}\right)_{j}^{m}+\hat{g}_{2} H_{m n}^{\prime l k}\left(T_{10}\right)^{n i j}\left(T_{8}\right)_{[i l] k}\left(M_{8}\right)_{j}^{m}+\hat{g}_{3} H_{m n}^{\prime l k}\left(T_{10}\right)^{n i j}\left(T_{8}\right)_{[l k] i}\left(M_{8}\right)_{j}^{m} \\
& +\hat{\sigma}_{1} H_{l n}^{\prime l k}\left(T_{10}\right)^{n i j}\left(T_{8}\right)_{[i k] j}\left(M_{8}\right)_{m}^{m} \\
& +\hat{\lambda}_{1} H_{l n}^{\prime l k}\left(T_{10}\right)^{n i j}\left(T_{8}\right)_{[i m] j}\left(M_{8}\right)_{k}^{m} \\
& +\hat{\gamma}_{1} H_{l n}^{\prime l k}\left(T_{10}\right)^{n i j}\left(T_{8}\right)_{[i k] m}\left(M_{8}\right)_{j}^{m}+\hat{\gamma}_{2} H_{l n}^{\prime l k}\left(T_{10}\right)^{n i j}\left(T_{8}\right)_{[i m] k}\left(M_{8}\right)_{j}^{m}+\hat{\gamma}_{3} H_{l n}^{\prime l k}\left(T_{10}\right)^{n i j}\left(T_{8}\right)_{[m k] i}\left(M_{8}\right)_{j}^{m}
\end{aligned}
$$

where $H_{m n}^{\prime k l} \equiv V_{m n} V_{k l}^{*}$ containing the CKM factors.

The TDA amplitudes for the $T_{10} \rightarrow T_{8} P$ decays are given in Tab. I. Noted that $\hat{f}_{i}$ terms in Fig. 1 (f) related to 
the $T_{10} \rightarrow T_{8} M_{8}$ decays only contribute to $\Sigma^{* 0} \rightarrow p \pi^{-}, \Sigma^{* 0} \rightarrow n \pi^{0}$ and $\Sigma^{*+} \rightarrow p \pi^{0}$ processes. Comparing the SU(3) IRA amplitudes in Tab. VIII of Ref. [28] with the TDA amplitudes in Tab. I, we have the following relations

$$
\begin{aligned}
\bar{a}_{1}=\frac{\hat{a}_{1}+\hat{b}_{1}}{4}, & \bar{e}_{1}=\frac{\hat{a}_{1}-\hat{b}_{1}+2 \hat{\beta}_{1}}{4}, & \bar{b}_{2}=-\frac{\hat{G}_{2}}{4}, \\
\bar{c}_{3}+\bar{c}_{2}=\hat{D}_{2}=\hat{E}_{2}, & 2 \bar{c}_{1}+\bar{c}_{2}+\bar{c}_{3}=\frac{\hat{D}_{1}+\hat{E}_{1}}{2}, & 2 \bar{d}_{1}=-\hat{F}_{2}, \\
\bar{f}_{1}+\bar{f}_{2}=\frac{\hat{G}_{1}+\hat{\Gamma}_{1}}{2}=-\hat{F}_{1}, & \bar{f}_{1}=\bar{f}_{3}, & \hat{\Gamma}_{2}=0 .
\end{aligned}
$$

From Eq. (9) one can see that the parameters $\hat{G}_{2}, \hat{D}_{1}+\hat{E}_{1}, \hat{D}_{2}, \hat{E}_{2}$ and $\hat{F}_{2}$ terms in TDA amplitudes are related to $\bar{b}_{2}, \bar{c}_{1,2,3}$ or $\bar{d}_{1}$ terms in IRA amplitudes, which only appear in the Wilson coefficient suppressed $C_{+}$term, so we will neglect their contributions in following numerical analysis.

Using the amplitude relations listed in Tab. I and the experimental data of $\mathcal{B}\left(\Omega \rightarrow \Xi^{0} \pi^{-}, \Xi^{-} \pi^{0}, \Lambda^{0} K^{-}\right)$, we obtain all branching ratio predictions for relevant $\Xi^{*-}, \Xi^{* 0}$ and $\Sigma^{*-}$ decays, which are listed in the last column of Tab. II. Noted that the mass difference in the amplitudes similar to Eq. (7) is also considered. In Tab. II, one can see that $\mathcal{B}\left(\Omega \rightarrow \Xi^{*-} \pi^{0}, \Xi^{* 0} \pi^{-}, \Sigma^{* 0} K^{-}\right)$are on the order of $\mathcal{O}\left(10^{-2}-10^{-1}\right)$, nevertheless, all branching ratios of $\Xi^{*}$ and $\Sigma^{*}$ weak decays are on the order of $\mathcal{O}\left(10^{-14}-10^{-12}\right)$. The main reason of significant magnitude difference comes the different decay mechanisms between $\Omega$ decays and $\Xi^{*}\left(\Sigma^{*}\right)$ decays. $\Omega$ decays such as $\Omega \rightarrow \Xi^{*-} \pi^{0}, \Xi^{* 0} \pi^{-}, \Sigma^{* 0} K^{-}$ decay modes mainly through weak interactions, and its life time $\tau_{\Omega} \approx 0.821 \times 10^{-10} s$. But $\Xi^{*}$ and $\Sigma^{*}$ decay mainly

\begin{tabular}{|c|c|c|c|}
\hline Observables & Tree & W-exchange & Penguin \\
\hline$A\left(\Omega^{-} \rightarrow \Xi^{0} \pi^{-}\right)$ & $\hat{a}_{1}$ & & $+\hat{\beta}_{1}$ \\
\hline$\sqrt{2} A\left(\Omega^{-} \rightarrow \Xi^{-} \pi^{0}\right)$ & $-\hat{b}_{1}$ & & $+\hat{\beta}_{1}$ \\
\hline$\sqrt{6} A\left(\Omega^{-} \rightarrow \Lambda^{0} K^{-}\right)$ & & $-\hat{G}_{1}$ & $-\hat{\Gamma}_{1}$ \\
\hline $3 \sqrt{2} A\left(\Xi^{*-} \rightarrow \Lambda^{0} \pi^{-}\right)$ & $3 \hat{a}_{1}$ & $-\hat{G}_{1}$ & $+3 \hat{\beta}_{1}-\hat{\Gamma}_{1}$ \\
\hline$\sqrt{6} A\left(\Xi^{*-} \rightarrow \Sigma^{0} \pi^{-}\right)$ & $\hat{a}_{1}$ & $+\hat{G}_{2}$ & $+\hat{\beta}_{1}+\hat{\Gamma}_{2}$ \\
\hline$\sqrt{6} A\left(\Xi^{*-} \rightarrow \Sigma^{-} \pi^{0}\right)$ & $-\hat{b}_{1}$ & & $+\hat{\beta}_{1}+\hat{\Gamma}_{2}$ \\
\hline$\sqrt{3} A\left(\Xi^{* 0} \rightarrow \Sigma^{+} \pi^{-}\right)$ & $\hat{a}_{1}$ & $+\hat{D}_{2}$ & $+\hat{\beta}_{1}$ \\
\hline $6 A\left(\Xi^{* 0} \rightarrow \Lambda^{0} \pi^{0}\right)$ & $-3 \hat{b}_{1}$ & $+\hat{D}_{1}+\hat{E}_{1}-\hat{G}_{1}$ & $+3 \hat{\beta}_{1}-\hat{\Gamma}_{1}$ \\
\hline $2 \sqrt{3} A\left(\Xi^{* 0} \rightarrow \Sigma^{0} \pi^{0}\right)$ & $\hat{b}_{1}$ & $-\hat{D}_{2}+\hat{E}_{2}+\hat{G}_{2}$ & $-\hat{\beta}_{1}+\hat{\Gamma}_{2}$ \\
\hline$\sqrt{3} A\left(\Xi^{* 0} \rightarrow \Sigma^{-} \pi^{+}\right)$ & & $-\hat{E}_{2}$ & $+\hat{\Gamma}_{2}$ \\
\hline$\sqrt{3} A\left(\Xi^{*-} \rightarrow n K^{-}\right)$ & & $\left(\hat{G}_{1}-\hat{G}_{2}\right) / 2$ & $-\left(\hat{\Gamma}_{2}-\hat{\Gamma}_{1}\right) / 2$ \\
\hline$\sqrt{3} A\left(\Sigma^{*-} \rightarrow n \pi^{-}\right)$ & $-\hat{a}_{1}$ & $+\left(\hat{G}_{1}-\hat{G}_{2}\right) / 2$ & $-\hat{\beta}_{1}-\left(\hat{\Gamma}_{2}-\hat{\Gamma}_{1}\right) / 2$ \\
\hline$\sqrt{6} A\left(\Sigma^{* 0} \rightarrow p \pi^{-}\right)$ & $-\hat{a}_{1}$ & $-\hat{D}_{2}+\left(\hat{F}_{1}+\hat{F}_{2}\right) / 2+\left(\hat{G}_{1}+\hat{G}_{2}\right) / 2$ & $-\hat{\beta}_{1}+\left(\hat{\Gamma}_{1}+\hat{\Gamma}_{2}\right) / 2$ \\
\hline $2 \sqrt{3} A\left(\Sigma^{* 0} \rightarrow n \pi^{0}\right)$ & $\hat{b}_{1}$ & $\left(\hat{D}_{2}-\hat{D}_{1}\right) / 2-\left(\hat{E}_{1}+\hat{E}_{2}\right) / 2-\hat{F}_{2}+\left(\hat{G}_{1}-\hat{G}_{2}\right) / 2$ & $-\hat{\beta}_{1}-\left(3 \hat{\Gamma}_{2}-\hat{\Gamma}_{1}\right) / 2$ \\
\hline$\sqrt{6} A\left(\Sigma^{*+} \rightarrow p \pi^{0}\right)$ & $\hat{b}_{1}$ & $-\left(\hat{D}_{2}+\hat{D}_{1}\right) / 2+\left(\hat{E}_{2}-\hat{E}_{1}\right) / 2+\left(\hat{F}_{1}+\hat{F}_{2}\right) / 2+\left(\hat{G}_{1}+\hat{G}_{2}\right) / 2$ & $-\hat{\beta}_{1}+\left(\hat{\Gamma}_{1}+\hat{\Gamma}_{2}\right) / 2$ \\
\hline
\end{tabular}

TABLE I: The TDA amplitudes of the $T_{10} \rightarrow T_{8} P$ decays under the $s \rightarrow u \bar{u} d$ transition. Noted that $\hat{X}_{1} \equiv \tilde{x}_{1}-\tilde{x}_{2}+2 \hat{x}_{3}$ and $\hat{X}_{2} \equiv \tilde{x}_{1}+\tilde{x}_{2}$ with $\hat{X}=\hat{G}, \hat{\Gamma}, \hat{D}, \hat{E}$ for $\hat{g}_{i}, \hat{\gamma}_{i}, \hat{d}_{i}, \hat{e}_{i}$, respectively. 
TABLE II: Branching ratios of the $T_{10} \rightarrow T_{8} P_{8}$ decays within $1 \sigma$ error under the $s \rightarrow u \bar{u} d$ transition. ${ }^{\dagger}$ denotes that the predictions depend on the relative phases.

\begin{tabular}{lcc}
\hline \hline Branching ratios & Exp. [1] & TDA \\
\hline $\mathcal{B}\left(\Omega^{-} \rightarrow \Xi^{0} \pi^{-}\right)\left(\times 10^{-2}\right)$ & $23.6 \pm 0.7$ & $23.6 \pm 0.7$ \\
$\mathcal{B}\left(\Omega^{-} \rightarrow \Xi^{-} \pi^{0}\right)\left(\times 10^{-2}\right)$ & $8.6 \pm 0.4$ & $8.6 \pm 0.4$ \\
$\mathcal{B}\left(\Omega^{-} \rightarrow \Lambda^{0} K^{-}\right)\left(\times 10^{-2}\right)$ & $67.8 \pm 0.7$ & $67.8 \pm 0.7$ \\
\hline $\mathcal{B}\left(\Xi^{*-} \rightarrow \Lambda^{0} \pi^{-}\right)\left(\times 10^{-12}\right)$ & $\ldots$ & $1.06 \pm 0.90^{\dagger}$ \\
$\mathcal{B}\left(\Xi^{*-} \rightarrow \Sigma^{0} \pi^{-}\right)\left(\times 10^{-14}\right)$ & $\ldots$ & $2.87 \pm 0.65$ \\
$\mathcal{B}\left(\Xi^{*-} \rightarrow \Sigma^{-} \pi^{0}\right)\left(\times 10^{-14}\right)$ & $\ldots$ & $2.14 \pm 0.52$ \\
$\mathcal{B}\left(\Xi^{* 0} \rightarrow \Sigma^{+} \pi^{-}\right)\left(\times 10^{-14}\right)$ & $\ldots$ & $5.96 \pm 0.58$ \\
$\mathcal{B}\left(\Xi^{* 0} \rightarrow \Lambda^{0} \pi^{0}\right)\left(\times 10^{-13}\right)$ & $\ldots$ & $5.02 \pm 4.06^{\dagger}$ \\
$\mathcal{B}\left(\Xi^{* 0} \rightarrow \Sigma^{0} \pi^{0}\right)\left(\times 10^{-14}\right)$ & $\ldots$ & $0.12 \pm 0.13$ \\
$\mathcal{B}\left(\Xi^{* 0} \rightarrow \Sigma^{-} \pi^{+}\right)$ & $\ldots$ & $2.03 \pm 1.24^{\dagger}$ \\
$\mathcal{B}\left(\Xi^{*-} \rightarrow n K^{-}\right)\left(\times 10^{-13}\right)$ & $\ldots$ & $6.14 \pm 1.31$ \\
$\mathcal{B}\left(\Sigma^{*-} \rightarrow n \pi^{-}\right)\left(\times 10^{-13}\right)$ & $\ldots$ & $2.20 \pm 1.48^{\dagger}$ \\
$\mathcal{B}\left(\Sigma^{* 0} \rightarrow p \pi^{-}\right)\left(\times 10^{-13}\right)$ & $\ldots$ & \\
$\mathcal{B}\left(\Sigma^{* 0} \rightarrow n \pi^{0}\right)\left(\times 10^{-13}\right)$ & $\ldots$ & \\
$\mathcal{B}\left(\Sigma^{*+} \rightarrow p \pi^{0}\right)\left(\times 10^{-13}\right)$ & $\ldots .41^{\dagger}$ \\
\hline
\end{tabular}

through strong interactions, such as $\Xi^{*} \rightarrow \Xi \pi$ as well as $\Sigma^{*} \rightarrow \Lambda \pi, \Sigma \pi$, and their life times are on the order of $10^{-23} s$. From Eq. (6), assuming all decay amplitudes are similar, the $\Xi^{*}$ and $\Sigma^{*}$ branching ratios are smaller than $\Omega$ decay branching ratios by about $10^{-12}$ orders. The similar reason for later $T_{10} \rightarrow T_{10}^{\prime} P_{8}$ decays listed in Tab. V.

\section{B. $\quad T_{10} \rightarrow T_{10}^{\prime} M_{8}$ weak decays}

For $T_{10} \rightarrow T_{10}^{\prime} M_{8}$ decays, their amplitudes are simple since both initial states $T_{10}$ and final states $T_{10}^{\prime}$ are all fully symmetric in flavor space. The TDA amplitudes of the $T_{10} \rightarrow T_{10}^{\prime} M_{8}$ decays are

$$
\begin{aligned}
A\left(T_{10} \rightarrow T_{10}^{\prime} M_{8}\right)^{T D A}= & \check{a} H_{m n}^{\prime k l}\left(T_{10}\right)^{n i j}\left(T_{10}\right)_{i j k}\left(M_{8}\right)_{l}^{m}+\check{b} H_{m n}^{\prime k l}\left(T_{10}\right)^{n i j}\left(T_{10}\right)_{i j l}\left(M_{8}\right)_{k}^{m} \\
+ & \check{c} H_{i n}^{\prime k l}\left(T_{10}\right)^{n i j}\left(T_{10}\right)_{k l j}\left(M_{8}\right)_{m}^{m}+\check{d} H_{i n}^{\prime k l}\left(T_{10}\right)^{n i j}\left(T_{10}\right)_{m k j}\left(M_{8}\right)_{l}^{m} \\
+ & \check{e} H_{i n}^{\prime k l}\left(T_{10}\right)^{n i j}\left(T_{10}\right)_{m l j}\left(M_{8}\right)_{k}^{m}+\check{f} H_{i n}^{\prime k l}\left(T_{10}\right)^{n i j}\left(T_{10}\right)_{l k m}\left(M_{8}\right)_{j}^{m} \\
+ & \check{g} H_{m n}^{\prime l k}\left(T_{10}\right)^{n i j}\left(T_{10}\right)_{i l k}\left(M_{8}\right)_{j}^{m}+\check{\sigma} H_{l n}^{\prime l k}\left(T_{10}\right)^{n i j}\left(T_{10}\right)_{k i j}\left(M_{8}\right)_{m}^{m} \\
& +\check{\lambda} H_{l n}^{\prime l k}\left(T_{10}\right)^{n i j}\left(T_{10}\right)_{m i j}\left(M_{8}\right)_{k}^{m}+\check{\gamma} H_{l n}^{\prime l k}\left(T_{10}\right)^{n i j}\left(T_{10}\right)_{i k m}\left(M_{8}\right)_{j}^{m} .
\end{aligned}
$$

And the TDA amplitudes for the $T_{10} \rightarrow T_{10}^{\prime} P_{8}$ decays are given in Tab. III.

Since the SU(3) IRA amplitudes of the $T_{10} \rightarrow T_{10}^{\prime} M_{8}$ decays have not been calculated in Ref. [28], we give them in 
TABLE III: The TDA amplitudes of the $T_{10} \rightarrow T_{10}^{\prime} P_{8}$ decays under the $s \rightarrow u \bar{u} d$ transition.

\begin{tabular}{|c|c|c|c|}
\hline Observables & Tree & W-exchange & Penguin \\
\hline$\sqrt{6} A\left(\Omega^{-} \rightarrow \Xi^{*-} \pi^{0}\right)$ & $\check{b}$ & & $-\check{\beta}$ \\
\hline$\sqrt{3} A\left(\Omega^{-} \rightarrow \Xi^{* 0} \pi^{-}\right)$ & $\check{a}$ & & $+\check{\beta}$ \\
\hline$\sqrt{6} A\left(\Omega^{-} \rightarrow \Sigma^{* 0} K^{-}\right)$ & & $\check{g}$ & $+\check{\gamma}$ \\
\hline $6 A\left(\Xi^{* 0} \rightarrow \Sigma^{* 0} \pi^{0}\right)$ & $\check{b}$ & $-\check{d}+\check{e}+\check{g}$ & $-\check{\beta}+\check{\gamma}$ \\
\hline $3 A\left(\Xi^{* 0} \rightarrow \Sigma^{*+} \pi^{-}\right)$ & $\check{a}$ & $+\check{d}$ & $+\check{\beta}$ \\
\hline $3 A\left(\Xi^{* 0} \rightarrow \Delta^{+} K^{-}\right)$ & & $\check{f}+\check{g}$ & $+\check{\gamma}$ \\
\hline $3 A\left(\Xi^{* 0} \rightarrow \Sigma^{*-} \pi^{+}\right)$ & & $\check{e}$ & $+\check{\gamma}$ \\
\hline $3 A\left(\Xi^{* 0} \rightarrow \Delta^{0} K^{0}\right)$ & & $\check{f}$ & $\check{\gamma}$ \\
\hline $3 \sqrt{2} A\left(\Xi^{*-} \rightarrow \Sigma^{*-} \pi^{0}\right)$ & $\check{b}$ & & $-\check{\beta}-\check{\gamma}$ \\
\hline $3 \sqrt{2} A\left(\Xi^{*-} \rightarrow \Sigma^{* 0} \pi^{-}\right)$ & $\check{a}$ & $+\check{g}$ & $+\check{\beta}+\check{\gamma}$ \\
\hline $3 A\left(\Xi^{*-} \rightarrow \Delta^{0} K^{-}\right)$ & & $\check{g}$ & $+\check{\gamma}$ \\
\hline$\sqrt{6} A\left(\Sigma^{*-} \rightarrow \Delta^{-} \pi^{0}\right)$ & $\breve{b}$ & & $-\check{\beta}-\check{\gamma}$ \\
\hline $3 A\left(\Sigma^{*-} \rightarrow \Delta^{0} \pi^{-}\right)$ & $\check{a}$ & $+\check{g}$ & $+\check{\beta}+\check{\gamma}$ \\
\hline $3 \sqrt{2} A\left(\Sigma^{*+} \rightarrow \Delta^{+} \pi^{0}\right)$ & $\check{b}$ & $-\check{d}+\check{e}+\check{f}+\check{g}$ & $-\check{\beta}+\check{\gamma}$ \\
\hline$\sqrt{3} A\left(\Sigma^{*+} \rightarrow \Delta^{++} \pi^{-}\right)$ & $\check{a}$ & $+\check{d}$ & $+\check{\beta}$ \\
\hline $6 A\left(\Sigma^{* 0} \rightarrow \Delta^{0} \pi^{0}\right)$ & $\check{b}$ & $-\check{d}+\check{e}-\check{f}+\check{g}$ & $-\check{\beta}$ \\
\hline $3 \sqrt{2} A\left(\Sigma^{* 0} \rightarrow \Delta^{+} \pi^{-}\right)$ & $\check{a}$ & $+\check{d}+\check{f}+\check{g}$ & $+\check{\beta}+\check{\gamma}$ \\
\hline
\end{tabular}

this work. The IRA amplitudes of the $T_{10} \rightarrow T_{10}^{\prime} M_{8}$ decays are

$$
\begin{aligned}
A\left(T_{10} \rightarrow T_{10}^{\prime} M_{8}\right)^{I R A}= & \ddot{a} H(4)_{m}^{l k}\left(T_{10}\right)^{i j n}\left(T_{10}\right)_{i j k}\left(M_{8}\right)_{l}^{m}+\ddot{b} H(4)_{m}^{l k}\left(T_{10}\right)^{i j n}\left(T_{10}\right)_{i l k}\left(M_{8}\right)_{j}^{m} \\
+ & \ddot{c} H(4)_{j}^{l k}\left(T_{10}\right)^{i j n}\left(T_{10}\right)_{i m k}\left(M_{8}\right)_{l}^{m}+\ddot{d} H(4)_{j}^{l k}\left(T_{10}\right)^{i j n}\left(T_{10}\right)_{m l k}\left(M_{8}\right)_{i}^{m} \\
+ & \ddot{e} H(\overline{2})^{k}\left(T_{10}\right)^{i j n}\left(T_{10}\right)_{i j m}\left(M_{8}\right)_{k}^{m}+\ddot{f} H(\overline{2})^{k}\left(T_{10}\right)^{i j n}\left(T_{10}\right)_{k i m}\left(M_{8}\right)_{j}^{m},
\end{aligned}
$$

where the non-zore current-current operators in $\mathrm{SU}(2)$ flavor space are $H(4)_{1}^{12}=H(4)_{1}^{21}=\frac{1}{3}$ and $H(\overline{2})^{2}=1$, small penguin contributions of $H(4)_{2}^{22}=-\frac{1}{3}$ are ignored in our analysis. And the concrete amplitudes for the $T_{10} \rightarrow T_{10}^{\prime} P_{8}$ decays are given in Tab. IV.

Comparing the TDA amplitudes in Tab. III and the IRA amplitudes in Tab. IV, we find that they match consistently in the $T_{10} \rightarrow T_{10}^{\prime} M_{8}$ decays, and the relations between the two sets of amplitudes are

$$
\begin{aligned}
& \ddot{a}+\ddot{e}=\check{a}+\check{\beta}, \quad 2 \ddot{a}-\ddot{a}^{\prime}=\check{a}+\check{b}, \quad 2 \ddot{b}+\ddot{f}=\check{g}, \\
& \ddot{c}=\check{d}=\check{e}, \quad \ddot{d}=\frac{1}{2} \check{f}, \\
& \ddot{f}=-\ddot{b}^{\prime}, \quad \check{\gamma}=0 \text {. }
\end{aligned}
$$


TABLE IV: The SU(3) IRA amplitudes of the $T_{10} \rightarrow T_{10}^{\prime} P_{8}$ decays under the $s \rightarrow u \bar{u} d$ transition. Noted that $\ddot{a}^{\prime}$ and $\ddot{b}^{\prime}$ denote $\ddot{a}$ and $\ddot{b}$ terms in $H(4)_{2}^{22}$, respectively.

\begin{tabular}{|c|c|c|c|}
\hline Observables & $H(4)_{1}^{12}=\frac{1}{3}$ & $H(4)_{2}^{22}=-\frac{1}{3}$ & $H(\overline{2})^{2}=1$ \\
\hline$\sqrt{6} A\left(\Omega^{-} \rightarrow \Xi^{*-} \pi^{0}\right)$ & $\ddot{a}$ & $-\ddot{a}^{\prime}$ & $-\ddot{e}$ \\
\hline$\sqrt{3} A\left(\Omega^{-} \rightarrow \Xi^{* 0} \pi^{-}\right)$ & $\ddot{a}$ & & $+\ddot{e}$ \\
\hline$\sqrt{6} A\left(\Omega^{-} \rightarrow \Sigma^{* 0} K^{-}\right)$ & $2 \ddot{b}$ & & $+\ddot{f}$ \\
\hline $6 A\left(\Xi^{* 0} \rightarrow \Sigma^{* 0} \pi^{0}\right)$ & $\ddot{a}+2 \ddot{b}$ & $-\ddot{a}^{\prime}$ & $-\ddot{e}+\ddot{f}$ \\
\hline $3 A\left(\Xi^{* 0} \rightarrow \Sigma^{*+} \pi^{-}\right)$ & $\ddot{a}+\ddot{c}$ & & $+\ddot{e}$ \\
\hline $3 A\left(\Xi^{* 0} \rightarrow \Delta^{+} K^{-}\right)$ & $2 \ddot{b}+2 \ddot{d}$ & & $+\ddot{f}$ \\
\hline $3 A\left(\Xi^{* 0} \rightarrow \Sigma^{*-} \pi^{+}\right)$ & $\ddot{c}$ & $+\ddot{b}^{\prime}$ & $+\ddot{f}$ \\
\hline $3 A\left(\Xi^{* 0} \rightarrow \Delta^{0} K^{0}\right)$ & $2 \ddot{d}$ & $+\ddot{b}^{\prime}$ & $\ddot{f}$ \\
\hline $3 \sqrt{2} A\left(\Xi^{*-} \rightarrow \Sigma^{*-} \pi^{0}\right)$ & $\ddot{a}$ & $-\ddot{a}^{\prime}-\ddot{b}^{\prime}$ & $-\ddot{e}-\ddot{f}$ \\
\hline $3 \sqrt{2} A\left(\Xi^{*-} \rightarrow \Sigma^{* 0} \pi^{-}\right)$ & $\ddot{a}+2 \ddot{b}$ & & $+\ddot{e}+\ddot{f}$ \\
\hline $3 A\left(\Xi^{*-} \rightarrow \Delta^{0} K^{-}\right)$ & $2 \ddot{b}$ & & $+\ddot{f}$ \\
\hline$\sqrt{6} A\left(\Sigma^{*-} \rightarrow \Delta^{-} \pi^{0}\right)$ & $\ddot{a}$ & $-\ddot{a}^{\prime}-\ddot{b}^{\prime}$ & $-\ddot{e}-\ddot{f}$ \\
\hline $3 A\left(\Sigma^{*-} \rightarrow \Delta^{0} \pi^{-}\right)$ & $\ddot{a}+2 \ddot{b}$ & & $+\ddot{e}+\ddot{f}$ \\
\hline $3 \sqrt{2} A\left(\Sigma^{*+} \rightarrow \Delta^{+} \pi^{0}\right)$ & $\ddot{a}+2 \ddot{b}+2 \ddot{d}$ & $-\ddot{a}^{\prime}$ & $-\ddot{e}+\ddot{f}$ \\
\hline$\sqrt{3} A\left(\Sigma^{*+} \rightarrow \Delta^{++} \pi^{-}\right)$ & $\ddot{a}+\ddot{c}$ & & $+\ddot{e}$ \\
\hline $6 A\left(\Sigma^{* 0} \rightarrow \Delta^{0} \pi^{0}\right)$ & $\ddot{a}+2 \ddot{b}-2 \ddot{d}$ & $-\ddot{a}^{\prime}-\ddot{b}^{\prime}$ & $-\ddot{e}$ \\
\hline $3 \sqrt{2} A\left(\Sigma^{* 0} \rightarrow \Delta^{+} \pi^{-}\right)$ & $\ddot{a}+2 \ddot{b}+\ddot{c}+2 \ddot{d}$ & & $+\ddot{e}+\ddot{f}$ \\
\hline
\end{tabular}

From the Tab. III, we have the following amplitude relations

$$
\begin{aligned}
A\left(\Omega^{-} \rightarrow \Xi^{*-} \pi^{0}\right) & =\sqrt{3} A\left(\Xi^{*-} \rightarrow \Sigma^{*-} \pi^{0}\right)=\sqrt{6} A\left(\Sigma^{*-} \rightarrow \Delta^{-} \pi^{0}\right), \\
\sqrt{2} A\left(\Omega^{-} \rightarrow \Sigma^{* 0} K^{-}\right)=\sqrt{3} A\left(\Xi^{*-} \rightarrow \Delta^{0} K^{-}\right), & \\
\sqrt{6} A\left(\Xi^{*-} \rightarrow \Sigma^{* 0} \pi^{-}\right)=\sqrt{3} A\left(\Sigma^{*-} \rightarrow \Delta^{0} \pi^{-}\right) & =A\left(\Omega^{-} \rightarrow \Xi^{* 0} \pi^{-}\right)+\sqrt{2} A\left(\Omega^{-} \rightarrow \Sigma^{* 0} K^{-}\right) \\
& =A\left(\Omega^{-} \rightarrow \Xi^{* 0} \pi^{-}\right)+\sqrt{2} A\left(\Xi^{*-} \rightarrow \Delta^{0} K^{-}\right),
\end{aligned}
$$

where we have used $\check{\gamma}=0$ and $-\check{d}+\check{e}=0$.

The W-exchange contributions in $T_{10} \rightarrow T_{10}^{\prime} M_{8}$ decays are strongly suppressed since the contraction of the flavor antisymmetric current-current operator with a flavor symmetric final state configuration is zero by Körner, Pati, Woo (KPW) theorem $[64,65]$. If we also ignore the $\mathrm{W}$-exchange contributions in $T_{10} \rightarrow T_{10}^{\prime} M_{8}$ decays, we can obtain more simple relations between the amplitudes from Tab. III. Ignoring the W-exchange contributions and in terms of the central values of the input parameters, one may get the ratios of their branching ratios, which are listed in the second and forth columns of Tab. V. In the second and forth columns of Tab. V, we take $\mathcal{B}\left(\Omega^{-} \rightarrow \Xi^{*-} \pi^{0}\right)$ and $\mathcal{B}\left(\Omega^{-} \rightarrow \Xi^{* 0} \pi^{-}\right)$as a unit for $\mathcal{B}\left(T_{10} \rightarrow T_{10}^{\prime} \pi^{0}\right)$ and $\mathcal{B}\left(T_{10} \rightarrow T_{10}^{\prime} \pi^{-}\right)$, respectively. 
TABLE V: Branching ratios of the $T_{10} \rightarrow T_{10}^{\prime} P_{8}$ decays under the $s \rightarrow u \bar{u} d$ transition. The ratios of the branching ratios are obtained in terms of the central values of the input parameters, and the upper limits of $\mathcal{B}\left(T_{10} \rightarrow T_{10}^{\prime} \pi^{-}\right)$are obtained with the $1 \sigma$ error of input parameters.

\begin{tabular}{|c|c|c|c|c|}
\hline Decay modes & $\frac{\mathcal{B}\left(T_{10} \rightarrow T_{10}^{\prime} \pi^{0}\right)}{\mathcal{B}\left(\Omega^{-} \rightarrow \Xi^{*-} \pi^{0}\right)}$ & Decay modes & $\frac{\mathcal{B}\left(T_{10} \rightarrow T_{10}^{\prime} \pi^{-}\right)}{\mathcal{B}\left(\Omega^{-} \rightarrow \Xi^{* 0} \pi^{-}\right)}$ & Upper Limits of $\mathcal{B}\left(T_{10} \rightarrow T_{10}^{\prime} \pi^{-}\right)$ \\
\hline$\Omega^{-} \rightarrow \Xi^{*-} \pi^{0}$ & 1 & $\Omega^{-} \rightarrow \Xi^{* 0} \pi^{-}$ & 1 & $7 \times 10^{-5}$ \\
\hline$\Xi^{* 0} \rightarrow \Sigma^{* 0} \pi^{0}$ & $1.89 \times 10^{-12}$ & $\Xi^{* 0} \rightarrow \Sigma^{*+} \pi^{-}$ & $7.87 \times 10^{-12}$ & $2.13 \times 10^{-15}$ \\
\hline$\Xi^{*-} \rightarrow \Sigma^{*-} \pi^{0}$ & $3.35 \times 10^{-12}$ & $\Xi^{*-} \rightarrow \Sigma^{* 0} \pi^{-}$ & $5.08 \times 10^{-12}$ & $1.47 \times 10^{-15}$ \\
\hline$\Sigma^{*-} \rightarrow \Delta^{-} \pi^{0}$ & $5.16 \times 10^{-12}$ & $\Sigma^{*-} \rightarrow \Delta^{0} \pi^{-}$ & $3.98 \times 10^{-12}$ & $1.15 \times 10^{-15}$ \\
\hline$\Sigma^{*+} \rightarrow \Delta^{+} \pi^{0}$ & $1.27 \times 10^{-12}$ & $\Sigma^{*+} \rightarrow \Delta^{++} \pi^{-}$ & $7.78 \times 10^{-12}$ & $2.27 \times 10^{-15}$ \\
\hline$\Sigma^{* 0} \rightarrow \Delta^{0} \pi^{0}$ & $6.95 \times 10^{-13}$ & $\Sigma^{* 0} \rightarrow \Delta^{+} \pi^{-}$ & $1.46 \times 10^{-12}$ & $4.57 \times 10^{-16}$ \\
\hline
\end{tabular}

Using the experimental upper limit $\mathcal{B}\left(\Omega^{-} \rightarrow \Xi^{* 0} \pi^{-}\right)<7 \times 10^{-5}$ at $90 \%$ CL [1] and considering the $1 \sigma$ error of relevant input parameters, we may predict the upper limits of $\mathcal{B}\left(T_{10} \rightarrow T_{10}^{\prime} \pi^{-}\right)$, which are listed in the last columns of Tab. V. We can see that all branching ratios are very tiny and they can not be measured in recent experiments such as BESIII and LHCb.

\section{C. $\quad T_{8} \rightarrow T_{8}^{\prime} M_{8}$ weak decays}

Topological diagrams for $T_{8} \rightarrow T_{8}^{\prime} M_{8}$ are also displayed in Fig. 1. Since both initial state $T_{8}$ and final state $T_{8}^{\prime}$ are all not fully symmetric or antisymmetric in flavor space, there are many amplitudes corresponding to one topological diagram, as a result, 92 amplitudes for the $T_{8} \rightarrow T_{8}^{\prime} M_{8}$ decays correspond to 10 topological diagrams in Fig. 1 . The TDA amplitudes of the $T_{8} \rightarrow T_{8}^{\prime} M_{8}$ decays can be constructed as

$$
\begin{aligned}
& A\left(T_{8} \rightarrow T_{8}^{\prime} M_{8}\right)^{T D A}=\tilde{a}_{1} H_{m n}^{k l}\left(T_{8}\right)^{[i j] n}\left(T_{8}\right)_{[i j] k}\left(M_{8}\right)_{l}^{m}+\tilde{a}_{2} H_{m n}^{k l}\left(T_{8}\right)^{[i j] n}\left(T_{8}\right)_{[i k] j}\left(M_{8}\right)_{l}^{m} \\
& +\tilde{a}_{3} H_{m n}^{k l}\left(T_{8}\right)^{[i n] j}\left(T_{8}\right)_{[i j] k}\left(M_{8}\right)_{l}^{m}+\tilde{a}_{4} H_{m n}^{k l}\left(T_{8}\right)^{[i n] j}\left(T_{8}\right)_{[i k] j}\left(M_{8}\right)_{l}^{m}+\tilde{a}_{5} H_{m n}^{k l}\left(T_{8}\right)^{[i n] j}\left(T_{8}\right)_{[k j] i}\left(M_{8}\right)_{l}^{m} \\
& +\tilde{b}_{1} H_{m n}^{k l}\left(T_{8}\right)^{[i j] n}\left(T_{8}\right)_{[i j] l}\left(M_{8}\right)_{k}^{m}+\tilde{b}_{2} H_{m n}^{k l}\left(T_{8}\right)^{[i j] n}\left(T_{8}\right)_{[i l] j}\left(M_{8}\right)_{k}^{m} \\
& +\tilde{b}_{3} H_{m n}^{k l}\left(T_{8}\right)^{[i n] j}\left(T_{8}\right)_{[i j] l}\left(M_{8}\right)_{k}^{m}+\tilde{b}_{4} H_{m n}^{k l}\left(T_{8}\right)^{[i n] j}\left(T_{8}\right)_{[i l] j}\left(M_{8}\right)_{k}^{m}+\tilde{b}_{5} H_{m n}^{k l}\left(T_{8}\right)^{[i n] j}\left(T_{8}\right)_{[l j] i}\left(M_{8}\right)_{k}^{m} \\
& +\tilde{c}_{1} H_{i n}^{l k}\left(T_{8}\right)^{[i j] n}\left(T_{8}\right)_{[j k] l}\left(M_{8}\right)_{m}^{m}+\tilde{c}_{2} H_{i n}^{l k}\left(T_{8}\right)^{[i j] n}\left(T_{8}\right)_{[j l] k}\left(M_{8}\right)_{m}^{m}+\tilde{c}_{3} H_{i n}^{l k}\left(T_{8}\right)^{[i j] n}\left(T_{8}\right)_{[k l] j}\left(M_{8}\right)_{m}^{m} \\
& +\tilde{c}_{4} H_{i n}^{l k}\left(T_{8}\right)^{[i n] j}\left(T_{8}\right)_{[j k] l}\left(M_{8}\right)_{m}^{m}+\tilde{c}_{5} H_{i n}^{l k}\left(T_{8}\right)^{[i n] j}\left(T_{8}\right)_{[j]] k}\left(M_{8}\right)_{m}^{m}+\tilde{c}_{6} H_{i n}^{l k}\left(T_{8}\right)^{[i n] j}\left(T_{8}\right)_{[k l] j}\left(M_{8}\right)_{m}^{m} \\
& +\tilde{c}_{7} H_{i n}^{l k}\left(T_{8}\right)^{[j n] i}\left(T_{8}\right)_{[j k] l}\left(M_{8}\right)_{m}^{m}+\tilde{c}_{8} H_{i n}^{l k}\left(T_{8}\right)^{[j n] i}\left(T_{8}\right)_{[j l] k}\left(M_{8}\right)_{m}^{m}+\tilde{c}_{9} H_{i n}^{l k}\left(T_{8}\right)^{[j n] i}\left(T_{8}\right)_{[k l] j}\left(M_{8}\right)_{m}^{m} \\
& +\tilde{d}_{1} H_{i n}^{l k}\left(T_{8}\right)^{[i j] n}\left(T_{8}\right)_{[j m] k}\left(M_{8}\right)_{l}^{m}+\tilde{d}_{2} H_{i n}^{l k}\left(T_{8}\right)^{[i j] n}\left(T_{8}\right)_{[j k] m}\left(M_{8}\right)_{l}^{m}+\tilde{d}_{3} H_{i n}^{l k}\left(T_{8}\right)^{[i j] n}\left(T_{8}\right)_{[k m] j}\left(M_{8}\right)_{l}^{m} \\
& +\tilde{d}_{4} H_{i n}^{l k}\left(T_{8}\right)^{[i n] j}\left(T_{8}\right)_{[j m] k}\left(M_{8}\right)_{l}^{m}+\tilde{d}_{5} H_{i n}^{l k}\left(T_{8}\right)^{[i n] j}\left(T_{8}\right)_{[j k] m}\left(M_{8}\right)_{l}^{m}+\tilde{d}_{6} H_{i n}^{l k}\left(T_{8}\right)^{[i n] j}\left(T_{8}\right)_{[k m] j}\left(M_{8}\right)_{l}^{m} \\
& +\tilde{d}_{7} H_{i n}^{l k}\left(T_{8}\right)^{[j n] i}\left(T_{8}\right)_{[j m] k}\left(M_{8}\right)_{l}^{m}+\tilde{d}_{8} H_{i n}^{l k}\left(T_{8}\right)^{[j n] i}\left(T_{8}\right)_{[j k] m}\left(M_{8}\right)_{l}^{m}+\tilde{d}_{9} H_{i n}^{l k}\left(T_{8}\right)^{[j n] i}\left(T_{8}\right)_{[k m] j}\left(M_{8}\right)_{l}^{m} \\
& +\tilde{e}_{1} H_{i n}^{l k}\left(T_{8}\right)^{[i j] n}\left(T_{8}\right)_{[j m] l}\left(M_{8}\right)_{k}^{m}+\tilde{e}_{2} H_{i n}^{l k}\left(T_{8}\right)^{[i j] n}\left(T_{8}\right)_{[j]] m}\left(M_{8}\right)_{k}^{m}+\tilde{e}_{3} H_{i n}^{l k}\left(T_{8}\right)^{[i j] n}\left(T_{8}\right)_{[l m] j}\left(M_{8}\right)_{k}^{m} \\
& +\tilde{e}_{4} H_{i n}^{l k}\left(T_{8}\right)^{[i n] j}\left(T_{8}\right)_{[j m] l}\left(M_{8}\right)_{k}^{m}+\tilde{e}_{5} H_{i n}^{l k}\left(T_{8}\right)^{[i n] j}\left(T_{8}\right)_{[j l] m}\left(M_{8}\right)_{k}^{m}+\tilde{e}_{6} H_{i n}^{l k}\left(T_{8}\right)^{[i n] j}\left(T_{8}\right)_{[l m] j}\left(M_{8}\right)_{k}^{m} \\
& +\tilde{e}_{7} H_{i n}^{l k}\left(T_{8}\right)^{[j n] i}\left(T_{8}\right)_{[j m] l}\left(M_{8}\right)_{k}^{m}+\tilde{e}_{8} H_{i n}^{l k}\left(T_{8}\right)^{[j n] i}\left(T_{8}\right)_{[j l] m}\left(M_{8}\right)_{k}^{m}+\tilde{e}_{9} H_{i n}^{l k}\left(T_{8}\right)^{[j n] i}\left(T_{8}\right)_{[l m] j}\left(M_{8}\right)_{k}^{m}
\end{aligned}
$$




$$
\begin{aligned}
& +\tilde{f}_{1} H_{i n}^{l k}\left(T_{8}\right)^{[i j] n}\left(T_{8}\right)_{[k l] m}\left(M_{8}\right)_{j}^{m}+\tilde{f}_{2} H_{i n}^{l k}\left(T_{8}\right)^{[i j] n}\left(T_{8}\right)_{[k m] l}\left(M_{8}\right)_{j}^{m}+\tilde{f}_{3} H_{i n}^{l k}\left(T_{8}\right)^{[i j] n}\left(T_{8}\right)_{[m l] k}\left(M_{8}\right)_{j}^{m} \\
& +\tilde{f}_{4} H_{i n}^{l k}\left(T_{8}\right)^{[i n] j}\left(T_{8}\right)_{[k l] m}\left(M_{8}\right)_{j}^{m}+\tilde{f}_{5} H_{i n}^{l k}\left(T_{8}\right)^{[i n] j}\left(T_{8}\right)_{[k m] l}\left(M_{8}\right)_{j}^{m}+\tilde{f}_{6} H_{i n}^{l k}\left(T_{8}\right)^{[i n] j}\left(T_{8}\right)_{[m l] k}\left(M_{8}\right)_{j}^{m} \\
& +\tilde{f}_{7} H_{i n}^{l k}\left(T_{8}\right)^{[j n] i}\left(T_{8}\right)_{[k l] m}\left(M_{8}\right)_{j}^{m}+\tilde{f}_{8} H_{i n}^{l k}\left(T_{8}\right)^{[j n] i}\left(T_{8}\right)_{[k m] l}\left(M_{8}\right)_{j}^{m}+\tilde{f}_{9} H_{i n}^{l k}\left(T_{8}\right)^{[j n] i}\left(T_{8}\right)_{[m l] k}\left(M_{8}\right)_{j}^{m} \\
& +\tilde{g}_{1} H_{m n}^{l k}\left(T_{8}\right)^{[i j] n}\left(T_{8}\right)_{[i k] l}\left(M_{8}\right)_{j}^{m}+\tilde{g}_{2} H_{m n}^{l k}\left(T_{8}\right)^{[i j] n}\left(T_{8}\right)_{[i l] k}\left(M_{8}\right)_{j}^{m}+\tilde{g}_{3} H_{m n}^{l k}\left(T_{8}\right)^{[i j] n}\left(T_{8}\right)_{[l k] i}\left(M_{8}\right)_{j}^{m} \\
& +\tilde{g}_{4} H_{m n}^{l k}\left(T_{8}\right)^{[i n] j}\left(T_{8}\right)_{[i k] l}\left(M_{8}\right)_{j}^{m}+\tilde{g}_{5} H_{m n}^{l k}\left(T_{8}\right)^{[i n] j}\left(T_{8}\right)_{[i l] k}\left(M_{8}\right)_{j}^{m}+\tilde{g}_{6} H_{m n}^{l k}\left(T_{8}\right)^{[i n] j}\left(T_{8}\right)_{[l k] i}\left(M_{8}\right)_{j}^{m} \\
& +\tilde{g}_{7} H_{m n}^{l k}\left(T_{8}\right)^{[j n] i}\left(T_{8}\right)_{[i k] l}\left(M_{8}\right)_{j}^{m}+\tilde{g}_{8} H_{m n}^{l k}\left(T_{8}\right)^{[j n] i}\left(T_{8}\right)_{[i l] k}\left(M_{8}\right)_{j}^{m}+\tilde{g}_{9} H_{m n}^{l k}\left(T_{8}\right)^{[j n] i}\left(T_{8}\right)_{[l k] i}\left(M_{8}\right)_{j}^{m} \\
& +\tilde{\sigma}_{1} H_{l n}^{l k}\left(T_{8}\right)^{[i j] n}\left(T_{8}\right)_{[i j] k}\left(M_{8}\right)_{m}^{m}+\tilde{\sigma}_{2} H_{l n}^{l k}\left(T_{8}\right)^{[i j] n}\left(T_{8}\right)_{[i k] j}\left(M_{8}\right)_{m}^{m} \\
& +\tilde{\sigma}_{3} H_{l n}^{l k}\left(T_{8}\right)^{[i n] j}\left(T_{8}\right)_{[i j] k}\left(M_{8}\right)_{m}^{m}+\tilde{\sigma}_{4} H_{l n}^{l k}\left(T_{8}\right)^{[i n] j}\left(T_{8}\right)_{[i k] j}\left(M_{8}\right)_{m}^{m}+\tilde{\sigma}_{5} H_{l n}^{l k}\left(T_{8}\right)^{[i n] j}\left(T_{8}\right)_{[k j] i}\left(M_{8}\right)_{m}^{m} \\
& +\tilde{\lambda}_{1} H_{l n}^{l k}\left(T_{8}\right)^{[i j] n}\left(T_{8}\right)_{[i j] m}\left(M_{8}\right)_{k}^{m}+\tilde{\lambda}_{2} H_{l n}^{l k}\left(T_{8}\right)^{[i j] n}\left(T_{8}\right)_{[i m] j}\left(M_{8}\right)_{k}^{m} \\
& +\tilde{\lambda}_{3} H_{l n}^{l k}\left(T_{8}\right)^{[i n] j}\left(T_{8}\right)_{[i j] m}\left(M_{8}\right)_{k}^{m}+\tilde{\lambda}_{4} H_{l n}^{l k}\left(T_{8}\right)^{[i n] j}\left(T_{8}\right)_{[i m] j}\left(M_{8}\right)_{k}^{m}+\tilde{\lambda}_{5} H_{l n}^{l k}\left(T_{8}\right)^{[i n] j}\left(T_{8}\right)_{[m j] i}\left(M_{8}\right)_{k}^{m} \\
& +\tilde{\gamma}_{1} H_{i n}^{l k}\left(T_{8}\right)^{[i j] n}\left(T_{8}\right)_{[i k] m}\left(M_{8}\right)_{j}^{m}+\tilde{\gamma}_{2} H_{i n}^{l k}\left(T_{8}\right)^{[i j] n}\left(T_{8}\right)_{[i m] k}\left(M_{8}\right)_{j}^{m}+\tilde{\gamma}_{3} H_{i n}^{l k}\left(T_{8}\right)^{[i j] n}\left(T_{8}\right)_{[m k] i}\left(M_{8}\right)_{j}^{m} \\
& +\tilde{\gamma}_{4} H_{i n}^{l k}\left(T_{8}\right)^{[i n] j}\left(T_{8}\right)_{[i k] m}\left(M_{8}\right)_{j}^{m}+\tilde{\gamma}_{5} H_{i n}^{l k}\left(T_{8}\right)^{[i n] j}\left(T_{8}\right)_{[i m] k}\left(M_{8}\right)_{j}^{m}+\tilde{\gamma}_{6} H_{i n}^{l k}\left(T_{8}\right)^{[i n] j}\left(T_{8}\right)_{[m k] i}\left(M_{8}\right)_{j}^{m} \\
& +\tilde{\gamma}_{7} H_{i n}^{l k}\left(T_{8}\right)^{[j n] i}\left(T_{8}\right)_{[i k] m}\left(M_{8}\right)_{j}^{m}+\tilde{\gamma}_{8} H_{i n}^{l k}\left(T_{8}\right)^{[j n] i}\left(T_{8}\right)_{[i m] k}\left(M_{8}\right)_{j}^{m}+\tilde{\gamma}_{9} H_{i n}^{l k}\left(T_{8}\right)^{[j n] i}\left(T_{8}\right)_{[m k] i}\left(M_{8}\right)_{j}^{m} .(14)
\end{aligned}
$$

From Eq. (14), we can get the TDA amplitudes of different decay modes. Since many parameters are not independent, we give the redefinitions

$$
\begin{aligned}
& X_{1} \equiv 2 \tilde{x}_{1}+2 \tilde{x}_{2}+2 \tilde{x}_{3}+\tilde{x}_{4}+\tilde{x}_{5}, \\
& X_{2} \equiv \tilde{x}_{4}-\tilde{x}_{5},
\end{aligned}
$$

where $X=A, B, \Sigma, \Lambda$ corresponding to $\tilde{a}_{i}, \tilde{b}_{i}, \tilde{\sigma}_{i}, \tilde{\lambda}_{i}$, respectively, and

$$
\begin{aligned}
Y_{1} & \equiv \tilde{y}_{1}+\tilde{y}_{3}+\tilde{y}_{4}+\tilde{y}_{6}, \\
Y_{2} & \equiv \tilde{y}_{4}+\tilde{y}_{6}+\tilde{y}_{7}+\tilde{y}_{9}, \\
Y_{3} & \equiv \tilde{y}_{4}+\tilde{y}_{5}+\tilde{y}_{7}+\tilde{y}_{8}, \\
Y_{4} & \equiv \tilde{y}_{2}-\tilde{y}_{3}+\tilde{y}_{5}-\tilde{y}_{6},
\end{aligned}
$$

with $Y=C, D, E, F, G, \Gamma$ for $\tilde{c}_{i}, \tilde{d}_{i}, \tilde{e}_{i}, \tilde{f}_{i}, \tilde{g}_{i}, \tilde{\gamma}_{i}$, respectively. And then the parameters related to $T_{8} \rightarrow T_{8}^{\prime} M_{8}$ decays studied in this paper are reduced to 26. The corresponding amplitudes are listed in Tab. VI. Even if neglecting 6 small penguin amplitudes, there still are 20 parameters. Since the parameters are too much, it's difficult for us to find many relations between different decay amplitudes. And there is only one relation for the decay amplitudes

$$
\sqrt{2} A\left(\Sigma^{+} \rightarrow p \pi^{0}\right)=A\left(\Sigma^{+} \rightarrow n \pi^{+}\right)+A\left(\Sigma^{-} \rightarrow n \pi^{-}\right)+\sqrt{2} A\left(\Sigma^{0} \rightarrow p \pi^{-}\right)+2 A\left(\Sigma^{0} \rightarrow n \pi^{0}\right),
$$

in which includes the amplitudes of the tree diagrams, the W-exchange diagrams and the penguin diagrams.

Many $T_{8} \rightarrow T_{8}^{\prime} P_{8}$ decays have been measured [1]

$$
\begin{aligned}
\mathcal{B}\left(\Sigma^{+} \rightarrow p \pi^{0}\right) & =(51.57 \pm 0.30) \times 10^{-2}, \\
\mathcal{B}\left(\Sigma^{+} \rightarrow n \pi^{+}\right) & =(48.31 \pm 0.30) \times 10^{-2}, \\
\mathcal{B}\left(\Sigma^{-} \rightarrow n \pi^{-}\right) & =(99.848 \pm 0.005) \times 10^{-2} \\
\mathcal{B}\left(\Lambda^{0} \rightarrow p \pi^{-}\right) & =(63.9 \pm 0.5) \times 10^{-2},
\end{aligned}
$$




$$
\begin{aligned}
\mathcal{B}\left(\Lambda^{0} \rightarrow n \pi^{0}\right) & =(35.8 \pm 0.5) \times 10^{-2}, \\
\mathcal{B}\left(\Xi^{-} \rightarrow \Lambda^{0} \pi^{-}\right) & =(99.887 \pm 0.035) \times 10^{-2} \\
\mathcal{B}\left(\Xi^{0} \rightarrow \Lambda^{0} \pi^{0}\right) & =(99.524 \pm 0.012) \times 10^{-2} .
\end{aligned}
$$

For not-yet-measured $\Sigma^{0} \rightarrow p \pi^{-}, n \pi^{0}$ decays, we may get the branching ratios in terms of the isospin relations [28]

$$
\begin{aligned}
\mathcal{B}\left(\Sigma^{0} \rightarrow p \pi^{-}\right) & =(4.82 \pm 0.50) \times 10^{-10}, \\
\mathcal{B}\left(\Sigma^{0} \rightarrow n \pi^{0}\right) & =(2.41 \pm 0.26) \times 10^{-10} .
\end{aligned}
$$

These measured branching ratios and obtained branching ratios from the isospin relations will help us to understand decay amplitudes of the tree diagrams and W-exchange diagrams. Using the central values in Eqs. (18-19) and the relation between the branching ratios and the moduli of the decay amplitudes in Eq. (6), one can backward obtain the ratios between the different moduli of decay amplitudes, which are given in the last column of Tab. VI. Noted that the ratios are obtained by using $\left|A\left(\Sigma^{-} \rightarrow n \pi^{-}\right)\right|$as a unit, in which we including the coefficients in front of the amplitudes in the first column of Tab. VI. For an example, in the second line and the last column of Tab. VI, the ratio 2.02 is obtained by using $\frac{\sqrt{2}\left|A\left(\Sigma^{+} \rightarrow p \pi^{0}\right)\right|}{\left|A\left(\Sigma^{-} \rightarrow n \pi^{-}\right)\right|}$, which is also equal to $\frac{\left|-B_{2}+D_{2}+E_{2}-E_{3}-F_{2}-G_{2}+2 \Lambda_{2}-\Gamma_{2}\right|}{\left|-A_{2}+G_{2}-G_{3}-2 \Lambda_{2}+\Gamma_{2}-\Gamma_{3}\right|}$. After ignoring the penguin contributions since they are small, we have the following remarks for Tab. VI.

- As shown in the third line, there is no tree diagram contribution to the $\Sigma^{+} \rightarrow n \pi^{+}$decay, nevertheless $\mid A\left(\Sigma^{+} \rightarrow\right.$ $\left.n \pi^{+}\right) \mid$may compare with others. So the $\mathrm{W}$-exchange contributions can be as large as some tree diagram contributions.

- For the tree diagram amplitudes shown in Fig. 1 (a-b), $A_{i} \propto C_{2}+C_{1} / N_{C} \approx 1.153$ and $B_{i} \propto C_{1}+C_{2} / N_{C} \approx$ -0.171 , i.e., $B_{i}$ are suppressed by the color-flavor factor. Comparing $\sqrt{6}\left|A\left(\Lambda^{0} \rightarrow p \pi^{-}\right)\right|$with $2 \sqrt{3}\left|A\left(\Lambda^{0} \rightarrow n \pi^{0}\right)\right|$

\begin{tabular}{|c|c|c|c|c|}
\hline Amplitudes & Tree & W-exchange & Penguin & Ratios of amplitude moduli \\
\hline$\sqrt{2} A\left(\Sigma^{+} \rightarrow p \pi^{0}\right)$ & $-B_{2}$ & $+D_{2}+E_{2}-E_{3}-F_{2}-G_{2}$ & $+2 \Lambda_{2}-\Gamma_{2}$ & 2.02 \\
\hline$A\left(\Sigma^{+} \rightarrow n \pi^{+}\right)$ & & $-E_{3}-F_{1}$ & $-\Gamma_{3}$ & 1.01 \\
\hline$A\left(\Sigma^{-} \rightarrow n \pi^{-}\right)$ & $-A_{2}$ & $+G_{2}-G_{3}$ & $-2 \Lambda_{2}+\Gamma_{2}-\Gamma_{3}$ & 1.00 \\
\hline$\sqrt{2} A\left(\Sigma^{0} \rightarrow p \pi^{-}\right)$ & $A_{2}$ & $+D_{3}-F_{2}-G_{2}$ & $+2 \Lambda_{2}-\Gamma_{2}$ & 2.02 \\
\hline $2 A\left(\Sigma^{0} \rightarrow n \pi^{0}\right)$ & $-B_{2}$ & $+D_{2}-D_{3}+E_{2}+F_{1}-G_{2}+G_{3}$ & $+2 \Lambda_{2}-\Gamma_{2}+2 \Gamma_{3}$ & 2.83 \\
\hline$\sqrt{6} A\left(\Lambda^{0} \rightarrow p \pi^{-}\right)$ & $-A_{1}$ & $\begin{array}{l}+2 D_{1}-D_{3}+2 D_{4} \\
-2 F_{1}+F_{2}-2 G_{1}+G_{2}\end{array}$ & $-2 \Lambda_{1}-2 \Gamma_{1}+\Gamma_{2}$ & 9.95 \\
\hline $2 \sqrt{3} A\left(\Lambda^{0} \rightarrow n \pi^{0}\right)$ & $-B_{1}$ & $\begin{array}{l}-D_{2}+D_{3}-2 D_{4}+2 E_{1}-E_{2} \\
+2 F_{1}-F_{3}+2 F_{4}-G_{2}+G_{3}-2 G_{4} \\
\end{array}$ & $+2 \Lambda_{1}+2 \Gamma_{1}-\Gamma_{2}$ & 14.17 \\
\hline$\sqrt{6} A\left(\Xi^{-} \rightarrow \Lambda^{0} \pi^{-}\right)$ & $\frac{1}{2} A_{1}+\frac{3}{2} A_{2}$ & $+G_{1}-2 G_{2}+G_{3}-G_{4}$ & $\begin{array}{l}+\left(\Lambda_{1}+3 \Lambda_{2}\right) \\
+\left(\Gamma_{1}-2 \Gamma_{2}+\Gamma_{3}-\Gamma_{4}\right)\end{array}$ & 9.66 \\
\hline $2 \sqrt{3} A\left(\Xi^{0} \rightarrow \Lambda^{0} \pi^{0}\right)$ & $\frac{1}{2} B_{1}+\frac{3}{2} B_{2}$ & $\begin{array}{l}-D_{1}+D_{4}-E_{1}+E_{4} \\
-G_{1}+2 G_{2}-G_{3}+G_{4} \\
\end{array}$ & $\begin{array}{l}-\left(\Lambda_{1}+3 \Lambda_{2}\right) \\
-\left(\Gamma_{1}-2 \Gamma_{2}+\Gamma_{3}-\Gamma_{4}\right)\end{array}$ & 15.24 \\
\hline
\end{tabular}
or comparing $\sqrt{6}\left|A\left(\Xi^{-} \rightarrow \Lambda^{0} \pi^{-}\right)\right|$with $2 \sqrt{3}\left|A\left(\Xi^{0} \rightarrow \Lambda^{0} \pi^{0}\right)\right|$, the formers included $A_{i}$ are smaller than the latter included $B_{i}$, so the W-exchange diagrams give large and even dominant contribution to relevant decay amplitudes.

TABLE VI: The TDA amplitudes of the $T_{8} \rightarrow T_{8}^{\prime} P_{8}$ decays under the $s \rightarrow u \bar{u} d$ transition. 


\section{SUMMARY}

In this work, we have analyzed the two-body nonleptonic decays of light baryon octet and decuplet by using the TDA under the SU(3) flavor symmetry. Comparing with the IRA, the TDA is more intuitive and gives a better understanding of dynamics.

We have found that the TDA and the IRA can match in the $T_{10} \rightarrow T_{8,10}^{\left({ }^{\prime}\right)} P_{8}$ weak decays. For the $T_{10} \rightarrow T_{8,10}^{\left({ }^{\prime}\right)} P_{8}$ weak decays, the TPA gives more simple predictions than the IRA since the W-exchange contributions are strongly suppressed. We have predicted all relevant decay branching ratios of the $T_{10} \rightarrow T_{8} P_{8}$ by using the TDA and also with the help of the IRA. For the $T_{10} \rightarrow T_{10}^{\prime} P_{8}$ decays, we have obtained some amplitude relations and the ratios of the branching ratios by using the TDA and the IRA, and then gotten the five upper limits of $\mathcal{B}\left(T_{10} \rightarrow T_{10}^{\prime} \pi^{-}\right)$by using the experimental upper limit of $\mathcal{B}\left(\Omega^{-} \rightarrow \Xi^{* 0} \pi^{-}\right)$. Nevertheless, since the lifetimes of $\Xi^{* 0,-}$ and $\Sigma^{* 0, \pm}$ are very small, all new branching ratio predictions, which are on the order of $\mathcal{O}\left(10^{-16}-10^{-12}\right)$, are too small to be searched in current experiments such as the BESIII and LHCb.

For the $T_{8} \rightarrow T_{8}^{\prime} P_{8}$ decays, since both initial states $T_{8}$ and final states $T_{8}^{\prime}$ are all not fully symmetric or antisymmetric in flavor space, these decays are quite complex in the theory, and the TPA gives more complex predictions than the IRA. Using some experiential branching ratios and other branching ratios obtained from the isospin relations, we analyze the contribution size of the tree diagrams and the W-exchange diagrams, and we have found that the $\mathrm{W}$ exchange diagrams give large and even dominant contributions to some decays.

\section{ACKNOWLEDGEMENTS}

The work was supported by the National Natural Science Foundation of China (Contract No. 11675137) and the Key Scientific Research Projects of Colleges and Universities in Henan Province (Contract No. 18A140029).

\section{References}

[1] M. Tanabashi et al. (Particle Data Group), Phys. Rev. D 98, 030001 (2018).

[2] H. B. Li, Front. Phys. (Beijing) 12, no. 5, 121301 (2017) [arXiv:1612.01775 [hep-ex]].

[3] I. I. Bigi, X. W. Kang and H. B. Li, Chin. Phys. C 42, no. 1, 013101 (2018)

[4] D. M. Asner et al., Int. J. Mod. Phys. A 24, S1 (2009) [arXiv:0809.1869 [hep-ex]].

[5] M. Ablikim et al. [BESIII Collaboration], Nature Physics (2019), https : //doi.org/10.1038/s41567 - 019 - $0494-8$.

[6] R. Aaij et al. [LHCb Collaboration], Phys. Rev. Lett. 120, no. 22, 221803 (2018) [arXiv:1712.08606 [hep-ex]].

[7] A. A. Alves Junior et al., JHEP 1905, 048 (2019), [arXiv:1808.03477 [hep-ex]].

[8] S. Weinberg, J. Phys. Conf. Ser. 196, 012002 (2009).

[9] N. Severijns, M. Beck and O. Naviliat-Cuncic, Rev. Mod. Phys. 78, 991 (2006) [nucl-ex/0605029].

[10] N. Cabibbo, Phys. Rev. Lett. 10, 531 (1963). 
[11] V. Cirigliano, M. Gonzalez-Alonso and M. L. Graesser, JHEP 1302, 046 (2013) [arXiv:1210.4553 [hep-ph]].

[12] H. M. Chang, M. González-Alonso and J. Martin Camalich, Phys. Rev. Lett. 114, no. 16, 161802 (2015) [arXiv:1412.8484 [hep-ph]].

[13] G. Altarelli, N. Cabibbo and L. Maiani, Phys. Lett. 57B, 277 (1975).

[14] M. J. Savage and R. P. Springer, Phys. Rev. D 42, 1527 (1990).

[15] M. J. Savage, Phys. Lett. B 257, 414 (1991).

[16] R. Arora, G. K. Sidana and M. P. Khanna, Phys. Rev. D 45, 4121 (1992).

[17] D. S. Du and D. X. Zhang, Phys. Rev. D 50, 2058 (1994).

[18] J. G. Korner, M. Kramer and D. Pirjol, Prog. Part. Nucl. Phys. 33, 787 (1994) [hep-ph/9406359].

[19] D. A. Egolf, R. P. Springer and J. Urban, Phys. Rev. D 68, 013003 (2003) [hep-ph/0211360].

[20] Y. K. Hsiao and C. Q. Geng, Phys. Rev. D 91, no. 11, 116007 (2015) [arXiv:1412.1899 [hep-ph]].

[21] M. Gronau and J. L. Rosner, Phys. Rev. D 89, no. 3, 037501 (2014) Erratum: [Phys. Rev. D 91, no. 11, 119902 (2015)] [arXiv:1312.5730 [hep-ph]].

[22] M. He, X. G. He and G. N. Li, Phys. Rev. D 92, no. 3, 036010 (2015) [arXiv:1507.07990 [hep-ph]].

[23] X. G. He and G. N. Li, Phys. Lett. B 750, 82 (2015) [arXiv:1501.00646 [hep-ph]].

[24] Y. K. Hsiao, C. F. Chang and X. G. He, Phys. Rev. D 93, no. 11, 114002 (2016) [arXiv:1512.09223 [hep-ph]].

[25] M. Gronau and J. L. Rosner, Phys. Rev. D 93, no. 3, 034020 (2016) [arXiv:1512.06700 [hep-ph]].

[26] C. D. Lü, W. Wang and F. S. Yu, Phys. Rev. D 93, no. 5, 056008 (2016) [arXiv:1601.04241 [hep-ph]].

[27] C. Q. Geng, Y. K. Hsiao, C. W. Liu and T. H. Tsai, Phys. Rev. D 99, no. 7, 073003 (2019) doi:10.1103/PhysRevD.99.073003 [arXiv:1810.01079 [hep-ph]].

[28] R. M. Wang, M. Z. Yang, H. B. Li and X. D. Cheng, Phys. Rev. D 100, no. 7, 076008 (2019) [arXiv:1906.08413 [hep-ph]].

[29] A. Dery, M. Ghosh, Y. Grossman and S. Schacht, arXiv:2001.05397 [hep-ph].

[30] D. Zeppenfeld, Z. Phys. C 8, 77 (1981).

[31] L. L. Chau and H. Y. Cheng, Phys. Rev. Lett. 56, 1655 (1986).

[32] L. L. Chau and H. Y. Cheng, Phys. Rev. D 36, 137 (1987) Addendum: [Phys. Rev. D 39, 2788 (1989)].

[33] M. J. Savage and M. B. Wise, Phys. Rev. D 39, 3346 (1989) Erratum: [Phys. Rev. D 40, 3127 (1989)].

[34] M. Gronau, O. F. Hernandez, D. London and J. L. Rosner, Phys. Rev. D 50, 4529 (1994) [hep-ph/9404283].

[35] M. Gronau, O. F. Hernandez, D. London and J. L. Rosner, Phys. Rev. D 52, 6374 (1995) [hep-ph/9504327].

[36] L. L. Chau, H. Y. Cheng, W. K. Sze, H. Yao and B. Tseng, Phys. Rev. D 43, 2176 (1991) Erratum: [Phys. Rev. D 58, $019902(1998)]$.

[37] H. Y. Cheng, C. W. Chiang and A. L. Kuo, Phys. Rev. D 91, no. 1, 014011 (2015) [arXiv:1409.5026 [hep-ph]].

[38] C. Wang, Q. A. Zhang, Y. Li and C. D. Lü, Eur. Phys. J. C 77, no. 5, 333 (2017) [arXiv:1701.01300 [hep-ph]].

[39] S. H. Zhou, Q. A. Zhang, W. R. Lyu and C. D. Lü, Eur. Phys. J. C 77, no. 2, 125 (2017) [arXiv:1608.02819 [hep-ph]].

[40] C. W. Chiang and Y. F. Zhou, JHEP 0612, 027 (2006) [hep-ph/0609128].

[41] S. H. Zhou, Y. B. Wei, Q. Qin, Y. Li, F. S. Yu and C. D. Lu, Phys. Rev. D 92, no. 9, 094016 (2015) [arXiv:1509.04060 [hep-ph]].

[42] C. W. Chiang and Y. F. Zhou, JHEP 0903, 055 (2009) [arXiv:0809.0841 [hep-ph]].

[43] S. Müller, U. Nierste and S. Schacht, Phys. Rev. D 92, no. 1, 014004 (2015) [arXiv:1503.06759 [hep-ph]].

[44] X. G. He and W. Wang, Chin. Phys. C 42, no. 10, 103108 (2018) [arXiv:1803.04227 [hep-ph]].

[45] X. G. He, J. Tandean and G. Valencia, JHEP 1907, 022 (2019) [arXiv:1903.01242 [hep-ph]].

[46] R. Flores-Mendieta, Phys. Rev. D 99, no. 9, 094033 (2019) [arXiv:1902.05602 [hep-ph]].

[47] F. J. Jiang and B. C. Tiburzi, Phys. Rev. D 77, 094506 (2008) [arXiv:0801.2535 [hep-lat]].

[48] J. Tandean, Phys. Rev. D 70, 076005 (2004) [hep-ph/0406274].

[49] B. Borasoy and E. Marco, Phys. Rev. D 67, 114016 (2003) [hep-ph/0306175]. 
[50] J. Tandean and G. Valencia, Phys. Rev. D 67, 056001 (2003) [hep-ph/0211165].

[51] M. D. Scadron and D. Tadic, J. Phys. G 27, 163 (2001) [hep-ph/0011328].

[52] A. Abd El-Hady and J. Tandean, Phys. Rev. D 61, 114014 (2000) [hep-ph/9908498].

[53] R. P. Springer, Phys. Lett. B 461, 167 (1999).

[54] J. Tandean and G. Valencia, Phys. Lett. B 451, 382 (1999) [hep-ph/9811376].

[55] B. Borasoy and B. R. Holstein, Eur. Phys. J. C 6, 85 (1999) [hep-ph/9805430].

[56] X. G. He and G. Valencia, Phys. Lett. B 409, 469 (1997) Erratum: [Phys. Lett. B 418, 443 (1998)] [hep-ph/9705462].

[57] E. E. Jenkins, Nucl. Phys. B 375, 561 (1992).

[58] J. M. Gaillard and G. Sauvage, Ann. Rev. Nucl. Part. Sci. 34, 351 (1984).

[59] T. N. Pham, Phys. Rev. D 87, no. 1, 016002 (2013) [arXiv:1210.3981 [hep-ph]].

[60] M. E. Carrillo-Serrano, I. C. Cloet and A. W. Thomas, Phys. Rev. C 90, no. 6, 064316 (2014) [arXiv:1409.1653 [nucl-th]].

[61] R. Flores-Mendieta, E. E. Jenkins and A. V. Manohar, Phys. Rev. D 58, 094028 (1998) [hep-ph/9805416].

[62] G. Buchalla, A. J. Buras and M. E. Lautenbacher, Rev. Mod. Phys. 68, 1125 (1996) [hep-ph/9512380].

[63] X. G. He, Y. J. Shi and W. Wang, arXiv:1811.03480 [hep-ph].

[64] J. G. Körner, Nucl. Phys. B 25, 282 (1971).

[65] J. C. Pati and C. H. Woo, Phys. Rev. D 3, 2920 (1971). 